\title{
Características morfológicas, morfométricas, merísticas y manejo de la primera alimentación de larvas de escalar altum (Pterophyllum altum) (Pellegrin, 1903)
}

\author{
Morphological, morphometric and meristic characteristics of \\ larvae of altum angelfish (Pterophyllum altum) (Pellegrin, 1903) \\ and management of its first feeding
}
Características morfológicas, morfométricas, merísticas y manejo da primeira alimentação de larvas de escalar altum (Pterophyllum altum) (Pellegrin, 1903)

\section{Alexander Torres-Tabares ${ }^{1 *}$; Yohana María Velasco-Santamaría ${ }^{2 *}$; Juan A. Ramírez-Merlano ${ }^{3 * *}$}

\author{
MVZ, Esp, MSc \\ $\mathrm{MV}, \mathrm{MSc}$, PhD \\ 3 Profesional en Acuicultura, MSc \\ * Grupo de Investigación en Biotecnología y Toxicología Acuática y Ambiental -BioTox, Escuela de Ciencias Animales \\ ** Grupo de Investigación sobre Reproducción y Toxicología de Organismos Acuáticos - GRITOX, Instituto de Acuicultura \\ Facultad de Ciencias Agropecuarias y Recursos Naturales, Universidad de los Llanos, Villavicencio, Meta - Colombia \\ Email: alexander.torres@unillanos.edu.co
}

Recibido: octubre 30 de 2014

Aceptado: noviembre 24 de 2014

\begin{abstract}
Resumen
El escalar altum ha sido una especie representativa de la ictiofauna suramericana por su amplia aceptación en el mercado de la acuariofilia, lo que lo convierte en una especie con gran potencial productivo. En el presente estudio se realizó una descripción morfológica, detalle de algunos parámetros merísticos de los primeros estadios de vida de larvas de $P$. altum y aspectos de su primera alimentación. Se utilizaron diez parejas de reproductores mantenidas en acuarios de $50 \mathrm{~L}$, con aireación y temperatura constante. Luego del proceso de reproducción se tomaron 15 larvas/h hasta las 75 horas poseclosión, (HPE) estas fueron fijadas en formol bufferado al $4 \%$ para posterior descripción de las características merísticas y morfométricas. Así mismo, se suministraron diferentes dietas luego de la reabsorción del saco vitelino, así: (T1) larvas alimentadas con nauplios de Artemia salina, (T2) cistos de Artemia salina decapsulados, y (T3) zooplancton silvestre para determinar algunos parámetros productivos. Morfológicamente, las larvas de $P$. altum tienen un cuerpo cilíndrico, redondeado, cola alargada y saco vitelino ovoide evidente hasta su desarrollo en postlarva. En cuanto a sus características morfométricas, presentaron una longitud total promedio de $4.64 \pm 0.5 \mathrm{~mm}$, una longitud estándar de $3.8 \pm 0.2 \mathrm{~mm}$, el diámetro del ojo de $0.49 \pm 0.2 \mathrm{~mm}$, así como una longitud de de cabeza $0.71 \pm 0.8 \mathrm{~mm}$ y la altura del cuerpo de $0.76 \pm 0.6 \mathrm{~mm}$ a las 75 HPE. Con el suministro de las diferentes dietas no se observaron diferencias estadísticas significativas ( $\mathrm{P}>0.05)$, en los pesos finales. Sin embargo, se observaron diferencias $(\mathrm{P}<0.05)$ en la tasa de crecimiento especifico y en la sobrevivencia. Como conclusión, las larvas de $P$. altum presentan un crecimiento acelerado hasta el segundo día de vida, debido a la disminución de la relación entre las características morfométricas. Por otro lado, el mejor desempeño productivo se observó en los
\end{abstract}


individuos alimentados con nauplios de artemia, constituyéndose en una importante alternativa como fuente de alimento durante la larvicultura de esta especie

Palabras clave: Alimento vivo, características morfológicas, crecimiento, peces, zooplancton.

\begin{abstract}
The altum angelfish is a representative species of the South American ichthyofauna due to it's wide acceptability in the aquariums' market, making it a species with great productive potential. In this study, a morphological description, detail of meristic parameters during its first life stages and aspects of first feeding were evaluated. Ten broodstock couples were kept in $50 \mathrm{~L}$ aquariums with aeration and constant temperature. After the breeding, 15 larvae/h were collected every hour until 75 hours post-hatching (HPH) and fixed in $4 \%$ buffered formalin for the analysis of the meristic and morphological characteristics. Likewise, different diets with Artemia salina nauplii (T1), capsulated Artemia salina cyst (T2) and wild zooplankton (T3) were offered to the larvae to determine some productive aspects. The $P$. altum larvae has cylindrical and rounded body, elongated tail and an ovoid yolk sac which is evident until it's development as post-larvae. Regarding its morphometric characteristics, the larvae had a total length of $4.64 \pm 0.5 \mathrm{~mm}$, standard length of $3.8 \pm 0.2 \mathrm{~mm}$, eye diameter of $0.49 \pm 0.2 \mathrm{~mm}$, head length of $0.71 \pm 0.8 \mathrm{~mm}$ and body height of $0.76 \pm 0.6 \mathrm{~mm}$ at $75 \mathrm{HPH}$. No significant differences between treatments were observed in the final weight $(\mathrm{P}>0.05)$; however, differences in the specific growth rate and in the survival were observed $(\mathrm{P}<0.05)$. The best productive performance was observed in individuals fed with Artemia nauplii. As a conclusion, $P$. altum larvae exhibit rapid growth until the second day of life, due to the decreased in the relationship between the morphometric characteristics. In the other hand, the best productive performance was observed in animal's brine shrimp nauplii individuals, becoming an important alternative food source during the larval rearing of this species.
\end{abstract}

Keywords: Live food, morphological characteristics, growth, fish, zooplankton.

\title{
Resumo
}

A anjo altum tem sido uma espécie de representação da fauna de peixes da América do Sul por causa de sua ampla aceitação no mercado do aquário, tornando-se uma espécie com grande potencial produtivo. No presente estudo a descrição morfológica detalhe de alguns parâmetros meristicos de fases iniciais de vida de larvas de $P$. altum e aspectos de sua primeira alimentação foi realizada. Dez pares de jogadores mantidos em aquários de 50 litros, com aeração e temperatura constante, então o processo de reprodução foram utilizadas 15 larvas / h foram tomadas até 75 horas pós-eclosão (HPE) e fixados em formalina tamponada a $4 \%$ para mais tarde descrição das características merísticos e morfométricos. Além disso, diferentes dietas após saco vitelino reabsorção foram fornecidos, bem como, (T1) larvas alimentadas náuplios de artémia (T2) de capsulated cistos camarão de água salgada e (T3) para determinar alguns parâmetros de produção do zooplâncton selvagens. As larvas de $P$. altum morfologicamente ter um cilíndrico, arredondado corpo, cauda longa e saco vitelino ovóide evidente até desenvolvimento pós-larval. Tal como para as suas características morfométricas apresentou um comprimento total médio de 4,64 $\pm 0.5 \mathrm{~mm}$, comprimento padrão de $3,8^{\circ} \pm 0,2 \mathrm{~mm}$, o diâmetro do olho de $0,49{ }^{\circ} \pm$ $0,2 \mathrm{~mm}$ e o comprimento de cabeça de $0,71 \pm 0,8 \mathrm{~mm}$ e uma altura corpo de 0,76 \pm 0,6 mm em 75 HPE. Com a oferta das diferentes dietas não foram estatisticamente diferentes $(P>0,05)$ nos pesos finais. No entanto, diferenças significativas $(P<0,05)$ na taxa de crescimento específico e sobrevivência foram observados. O melhor desempenho de crescimento foi observada nos animais alimentados com indivíduos de artémia. Em conclusão, as larvas de $P$. Altum a presentam rápido crescimento até o segundo dia de vida, por causa da diminuição da relação entre as características morfométricas. Por outro lado, o melhor desempenho produtivo foi observada nos animais alimentados indivíduos náuplios de artémia, tornando-se uma importante fonte alternativa de alimento durante a larvicultura desta espécie

Palavras chave: Alimento vivo, morfológicas, de crescimento, peixes, zooplâncton.

\section{Introducción}

El género Pterophyllum ha sido representativo de la ictio fauna suramericana, distribuido ampliamente en la cuenca del río Orinoco y el Amazonas Brasilero, Colombiano y Peruano; ríos que cuentan con aguas con temperaturas que oscilan entre 27 a $30^{\circ} \mathrm{C}$ y pH ácidos de 6.5 (Norman Jr. 1975; Axelrod y Walker, 2000; Pérez, et al., 2003; Abdolbaghian, et al., 2010).

Bajo la denominación de escalares se agrupan varias especies pertenecientes al género Pterophyllum spp y cuya sistemática es aún discutida (Agudelo, 2006). Dentro de estas denominaciones se encuentra el Pterophyllum altum, también conocido como escalare alto por las dimensiones en su altura, las cuales son superiores a las de cualquiera de las otras especies del género. Esta especie puede llegar a alcanzar hasta 18 $\mathrm{cm}$ de longitud y una mayor altura (Castro, 1985). Estas características lo hacen una especie muy apetecida para la acuariofilia de peces ornamentales. A pesar de su importancia comercial, son pocos los estudios encontrados con relación a su producción. La gran 
mayoría de estos toman como modelo de estudio la especie $P$. scalare debido a su mayor comercialización. En muchos casos, esta especie ha sido producida y comercializada de una manera empírica, ocasionando elevadas mortalidades, baja fertilidad, incidencia de enfermedades y lento crecimiento (Rodrigues y Fernandes, 2006; Zuanon et al., 2006).

Dentro de los aspectos más importantes en la acuicultura de peces ornamentales está la nutrición, especialmente durante las primeras etapas de vida (Sales y Janssens, 2003). La probabilidad que las larvas se alimenten luego de un proceso de reabsorción del saco vitelino depende, en gran medida, de la coincidencia entre el tamaño de la presa y el tamaño de la larva; apertura bucal, tipo de nado, capacidad visual y cantidad de alimento disponible por larva (Hamre et al., 2008). La larvicultura de peces se basa fundamentalmente en el suministro de artemia salina sin incorporar el uso de fuentes alimenticias de gran valor nutricional como el zooplancton, debido especialmente al desconocimiento que se tiene sobre la propia biología y fisiología de la especie (Halver, 1988; Guillaume et al., 2004). La descripción de la aparición y desarrollo de las características corporales externas y el estado de la morfología de las larvas, es importante para comprender el surgimiento de estructuras, con las respectivas ordenes cronológicas, que permiten en una larva una mayor autonomía de movimiento, alimentación y percepción del medio; consecuentemente, estas estructuras proporcionan una mayor sobrevivencia en el medio que se encuentran (Godinho et al., 2003).

En la acuicultura de peces ornamentales, factores como las deficiencias nutricionales y el alto costo de alimentos concentrados, han conducido a buscar alternativas para la alimentación en las primeras etapas de vida. Sin embargo, poco se ha estudiado sobre las preferencias alimenticias, calidad y aporte nutricional del alimento vivo; densidad alimenticia y características morfométricas (apertura bucal), las cuales influyen directamente sobre el desarrollo y la alimentación de las larvas (Erdogan y Olmez, 2009).

Teniendo en cuenta el desconocimiento de algunos aspectos biológicos del desarrollo inicial de la especie $P$. altum, el presente estudio tiene como objetivo contribuir con la caracterización morfométrica y merística, así como algunos aspectos de su primera alimentación, los cuales son importantes para comprender la estructura y dinámica alimenticia de esta especie.

\section{Materiales y métodos}

\section{Localización del área de estudio}

La investigación fue realizada en el laboratorio de peces ornamentales del Instituto de Acuicultura de la Universidad de los Llanos, ubicado en la vereda Barcelona, a $12 \mathrm{Km}$ de la ciudad de Villavicencio, departamento del Meta. Con una altitud de 418 metros sobre el nivel del mar, temperatura de $25^{\circ} \mathrm{C}$, precipitación de $4050 \mathrm{~mm}$ y humedad relativa del $75 \%$.

\section{Material biológico}

\section{Obtención de huevos y larvas}

Se utilizaron diez parejas adultas de la especie escalar altum (Pterophyllum altum) procedentes del stock de reproductores del Instituto de Acuicultura de la Universidad de los Llanos. El criterio de selección se basó en la formación de parejas y características externas reproductivas de las hembras, como abultamiento abdominal y enrojecimiento de la papila urogenital. Cada pareja se mantuvo en acuarios de $50 \mathrm{~L}$, con aireación y temperatura constante. En cada acuario se dispuso un tubo de PVC de $50 \mathrm{~cm}$ de largo, con una superficie lisa para facilitar el desove de la hembra y posterior fertilización por parte del macho. Seguidamente, los embriones fertilizados fueron trasladados a acuarios de $20 \mathrm{~L}$, donde permanecieron hasta su eclosión.

Durante la etapa larvaria, cada hora post-eclosión (HPE) y hasta las $75 \mathrm{HPE}$, se tomaron 15 larvas de $P$ altum que, de inmediato, fueron fijadas en formol buferado al $4 \%$ a temperatura ambiente, para el posterior registro y descripción de sus características merísticas y morfométricas.

\section{Descripción morfológica de la larva}

Para la descripción morfológica aparente, el material fijado fue transferido en agua destilada durante 24 horas para su rehidratación. El análisis morfológico consistió en la verificación macroscópica de las siguientes características: presencia y localización de pigmentación en el cuerpo (presencia y tipos de cromatóforos) y en el ojo (pigmentación en la retina), desplazamiento de la hendidura bucal, abertura del ano, formación de arcos branquiales y opérculo, esbozo de la vejiga gaseosa, reducción del saco vitelino y flexión del notocordio. 


\section{Características morfométricas}

Las características morfométricas evaluadas fueron:

- Longitud total (LT): desde el extremo del hocico hasta el extremo de la lámina de la aleta caudal.

- Longitud estándar (LS): desde el extremo del hocico hasta el extremo distal de la notocordia.

- Diámetro del ojo (DO): diámetro del ojo medido horizontalmente (Britski et al., 1988).

- Longitud de la cabeza (LC): distancia de la punta del hocico hasta la porción ósea posterior del opérculo (Britski et al., 1988).

- Altura del cuerpo (AC): distancia entre los extremos dorsal y ventral del cuerpo (Britski et al., 1988).

\section{Características merísticas}

Las características merísticas evaluadas fueron:

- Número total de miómeros (NM): (Nakatani et al., 2001).

- Número de miómeros pre-ano y pos-ano: número de miómeros anteriores (MPRE).

- Número de miómeros posteriores (MPOS) al ano (Nakatani et al., 2001).

La edad de las larvas se estableció en horas (h), a partir de la eclosión. El análisis fue realizado con la ayuda de una cámara de microfotografía (NIKON Digital, SIGHT DS-U1, Japón), acoplada a un microscopio de contraste de fase (NIKON, Japón, ECLIPSE E400) o a un estereoscopio (NIKON SMZ800, Japón). Todas las fotografías fueron almacenadas usando el software ACT-2U (NIKON, ACT-2U Imaging Software, Japón).

\section{Manejo de la primera alimentación}

Abertura máxima bucal (Amb): Para determinar la abertura máxima bucal (Amb) se hizo un seguimiento de la abertura de la boca, desde su inicio hasta que alcanzara la abertura máxima (Amb), considerando un ángulo de $90^{\circ}$, empleando la ecuación de Shirota (1970), así:

\section{Amb: LMSx $\sqrt{2}$}

Dónde Amb: Abertura máxima de la boca.

LMS: Longitud $(\mu \mathrm{m})$ máxima del maxilar superior.

\section{Alimentación y desarrollo corporal de larvas de escalar altum durante la fase de larvicultura}

Para la evaluación del desarrollo corporal y la sobrevivencia de las larvas fueron utilizados individuos que iniciaron su alimentación exógena. Las larvas fueron trasladadas a unidades experimentales, consistentes de recipientes en acrílico con un volumen efectivo de agua de $3 \mathrm{~L}$ y distribuidas en tres tratamientos de forma aleatoria, así: larvas alimentadas con nauplios de Artemia salina recién eclosionados, (Artemia High 5, Marca INVE) (T1), cistos de Artemia salina decapsulados (T2) y zooplancton silvestre, libre de predadores (300 a $600 \mu \mathrm{m})(\mathrm{T} 3)$. El zooplancton, conformado principalmente por cladóceros del genero Diaphanosoma $s p$, fue suministrado por el Laboratorio de Alimento Vivo del Instituto de Acuicultura de la Universidad de los Llanos.

Los diferentes tipos de alimentación fueron suministrados cuatro veces al día (7:00, 10:00, 15:00 y 19:00 h). Adicionalmente se ajustó la ración cada cuatro días, a una razón de 50, 100, 150 organismos por larva. Cada tratamiento contó con, mínimo, 3 unidades experimentales $(n=3)$. Dos horas después de cada alimentación se recambió el $20 \%$ del agua, por medio de sifoneo con el objetivo de retirar los organismos no consumidos y desechos.

Para evaluar el crecimiento, al inicio y final de la fase experimental, las postlarvas de cada tratamiento fueron pesadas en una balanza analítica (Explorer Pro OHAUS, Modelo EP214C) y medidas con un calibrador $(0.01 \pm \mathrm{SD})$, con el fin de determinar los siguientes parámetros productivos:

Ganancia en peso $(\mathbf{m g})$ : $\mathrm{GP}=\mathrm{Pf}-\mathrm{Pi}$, donde: $\mathrm{Pf}=\mathrm{Peso}$ final; $\mathrm{Pi}:=$ Peso inicial.

Ganancia en longitud $(\mathbf{m m}): G L=L f-L i$, donde: $L f=$ Longitud total final; $\mathrm{Li}=$ Longitud total inicial.

Tasa de Crecimiento Específico (\%/día): TCE = $((\mathrm{LnPmf}-\mathrm{LnPmi}) / \mathrm{t}){ }^{*} 100$, donde: $\mathrm{Pmi}=$ peso promedio inicial de las postlarvas (mg); $\mathrm{Pmf}=$ peso promedio final de las postlarvas (mg); $\mathrm{t}=$ tiempo de cultivo (días); $\mathrm{Ln}=$ logaritmo neperiano.

Sobrevivencia final (S): De cada unidad experimental, fueron calculados manualmente las post larvas vivas, al finalizar el ensayo.

Todos los días en cada recipiente, se determinaron parámetros físico-químicos como: temperatura $\left({ }^{\circ} \mathrm{C}\right)$, oxígeno disuelto $(\mathrm{mg} / \mathrm{l})$, \% de saturación del oxígeno, $\mathrm{pH}$, 
salinidad (\%) y conductividad ( $\mu \mathrm{s} / \mathrm{cm})$, utilizando una sonda multiparamétrica YSI (MPS YSI 556).

\section{Análisis Estadístico}

Los resultados fueron expresados como media \pm desviación estándar (DS). Para las variables morfométricas y merísticas se determinó la relación existente a través de un análisis de correlación de Pearson. La evaluación del desarrollo corporal y la sobrevivencia de las larvas fueron analizadas a través de estadística no paramétrica (datos no normales) mediante el test de KruskalWallis, seguido de la prueba de Dunn. En todos los casos, $\mathrm{P}<0,05$ fue utilizado como criterio estadístico para considerar diferencias significativas. Los procedimientos estadísticos fueron realizados empleando el software GraphPad versión 5.0 y SAS versión 9.2

\section{Resultados}

\section{Descripción morfológica de la larva}

Las características larvarias observadas en $P$. altum presentaron algunas diferencias marcadas, relacionadas con el estado de desarrollo larvario. En general, la larva presenta forma alargada, cuerpo cilíndrico, redondeado y cola alargada; ojos redondos, sin pigmentación; y un saco vitelino ovoide evidente desde sus estadios iniciales de vida, hasta su desarrollo en postlarva. El saco vitelino fue reabsorbido a las 75 HPE (Figura 1A).

Las larvas de un día de eclosión exhibieron pigmentación seriada y continua, a lo largo de la zona media de su cuerpo. Esas características se dan un poco más marcadas en larvas de dos y tres días de vida. También se observaron algunos primordios alargados los cuales formarán los filamentos branquiales. A partir del cuarto y quinto día se observaron los opérculos claramente abiertos y funcionales (Figura 1B). En su mayoría, las larvas de 72 HPE han reabsorbido en un $80 \%$ su saco vitelino y se encuentran listas para iniciar el consumo de alimento vivo. Sus ojos se encuentran totalmente pigmentados con una coloración oscura. En los estadios tardíos, su cuerpo es alargado y su cabeza pequeña. Así mismo, se observa un intestino largo y tubular. Otros detalles como la proyección de la boca y opérculos pueden ser apreciados, así como flexión del notocordio en los estadios de desarrollo tardío.

\section{Características morfométricas}

Los resultados de las mediciones morfométricas de larvas de P. altum (Figura 2) revelaron una LT promedio de $3.8 \mathrm{~mm}$, una LS de $4.64 \mathrm{~mm}$, el diámetro de ojo de $0.49 \mathrm{~mm}$ (DO), longitud de la cabeza $0.71 \mathrm{~mm}$ (LC) y la altura del cuerpo (AC) de $0.76 \mathrm{~mm}$ durante $75 \mathrm{HPE}$ (Tabla 1). Los cambios entre LT y LS de larvas de 0-3 días son proporcionales y presentan una relación alométrica lo cual es característico durante su desarrollo y crecimiento.

La disminución de la relación entre la LS y la AC se atribuye a un crecimiento más acelerado de algunos individuos a medida que aumenta la edad. La relación entre longitud estándar y altura se incrementó desde la eclosión (Edad 0) hasta el segundo día de vida de la larva (Edad II), y disminuyó para el tercer día de vida (Edad III) (Figura 3). Esta disminución en la altura de las larvas viene secuenciada de la reabsorción del saco vitelino, coincidiendo con la mayor mortalidad de larvas.
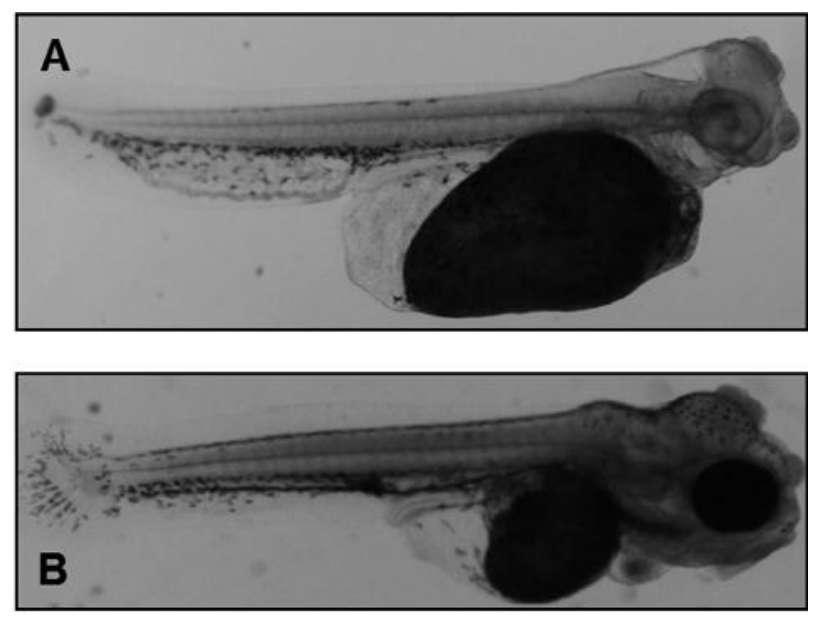

Figura 1. Larva de P. altum en diferentes horas pos-eclosión (HPE). A. 24 HPE (12X) B. 75 HPE (12X). 


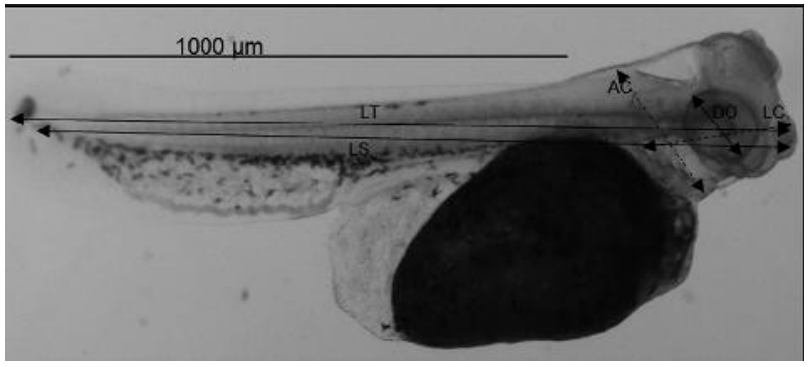

Figura 2. Características morfométricas $(\mu \mathrm{m})$ de larvas de $P$. altum de $24 \mathrm{HPE}(12 \mathrm{X})$.

$\mathbf{L T}=$ longitud total. $\mathbf{L S}=$ longitud estándar. $\mathbf{A C}=$ Altura del cuerpo. $\mathbf{D O}=$ diámetro del ojo. $\mathbf{L C}=$ Longitud de la cabeza.

Tabla 1. Características morfométricas $(\mu \mathrm{m})$ de larvas de escalar $P$. altum, de edad 0 , I y II correspondientes a larvas recién eclosionadas $(24$ h), de 48 h y 75 h pos-eclosión, respectivamente. Datos mostrados como promedio \pm desviación estándar.

\begin{tabular}{|c|c|c|c|}
\hline Medida & Edad 0 & Edad I & Edad II \\
\hline LT & $3601 \pm 1,10$ & $3907 \pm 0,53$ & $4079 \pm 0,64$ \\
\hline LS & $4064 \pm 1,05$ & $4857 \pm 0,55$ & $5026 \pm 0,61$ \\
\hline DO & $433 \pm 0,28$ & $480 \pm 0,25$ & $578 \pm 0,27$ \\
\hline LC & $694 \pm 1,35$ & $715 \pm 1,22$ & $744 \pm 1,13$ \\
\hline AC & $723 \pm 0,10$ & $761 \pm 0,11$ & $815 \pm 0,08$ \\
\hline
\end{tabular}

$\mathrm{LT}=$ Longitud total, $\mathrm{LS}=$ Longitud estándar $\mathrm{DO}=$ Diámetro del ojo, $\mathrm{LC}=$ Longitud de la cabeza y $\mathrm{AC}=$ Altura del cuerpo .

En el crecimiento de las larvas de escalares se pueden distinguir tres fases: la primera o inicial de crecimiento rápido (Edad 0-I), la segunda de crecimiento lento (Edad I-II) y la tercera de crecimiento negativo (Edad II-III). A medida que aumenta la edad, la relación entre longitud estándar y la altura se incrementa desde la eclosión (Edad 0) hasta el segundo día de vida de la larva (Edad II). Dicha relación disminuye para el tercer día de vida (Figura 3). Esta disminución en la altura de las larvas viene seguida de la reabsorción del saco vitelino, y coincide con la mayor mortalidad de larvas.

\section{Características merísticas}

Número total de miomeros (NM): Las larvas de $P$. altum mostraron en su longitud total un número entre de 75 a 89 miomeros que son más visibles a medida que se desarrollan las larvas. El número de miomeros antes del ano son aproximadamente 17 y posterior al ano alrededor de 22 (Figura 4 y 5).

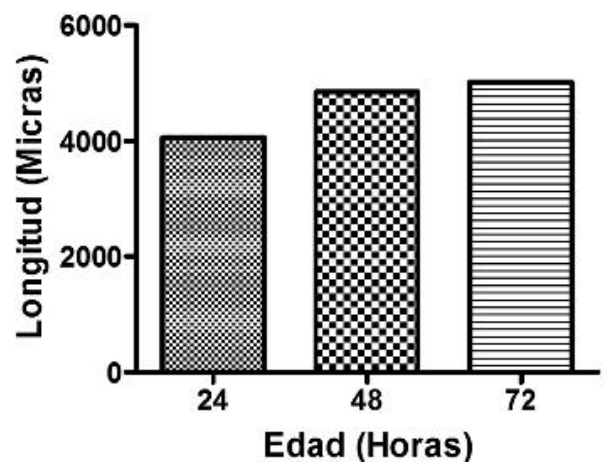

Figura 3. Crecimiento en larvas de P. altum de 0-3 días de vida (relación edad-longitud).

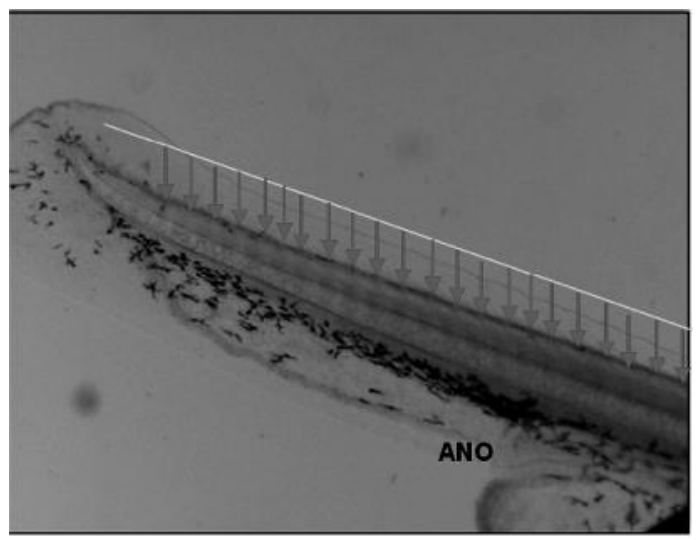

Figura 4. Parte posterior de la cola de una larva de $P$. altum de 75 HPE. Las flechas ilustran los miomeros posteriores al ano $(24 X)$.

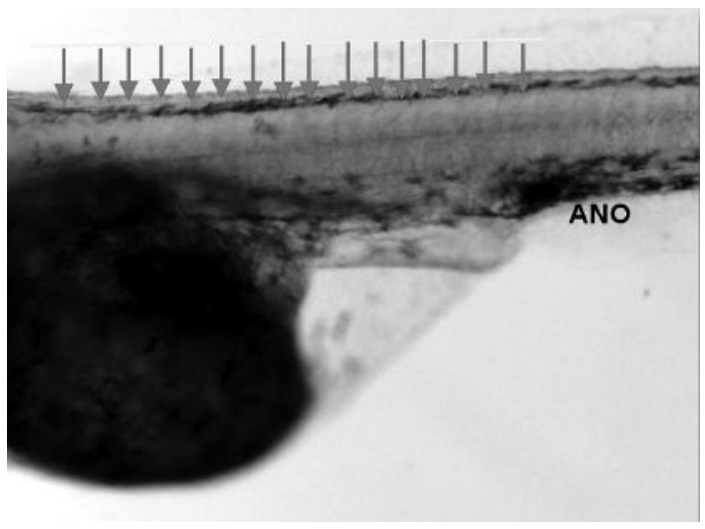

Figura 5. Parte Anterior de la cola de una larva de $P$. altum de $75 \mathrm{HPE}$. Las flechas ilustran los miomeros anteriores al ano (24X).

Manejo de la primera alimentación

\section{Abertura máxima bucal (Amb)}

Durante las 3 HPE comienzan a formarse los pliegues orales. Estos empiezan a separarse a las 12 HPE. La 
boca se abre y se cierra con movimientos fuertes y se comienzan a formar los dientes a las 24 HPE (Figura 6). A las 72 HPE aproximadas finaliza la reabsorción del saco vitelino e inicia la alimentación exógena con una abertura bucal de 0.5 a $1.0 \mathrm{~mm}$.

Los parámetros fisicoquímicos del agua se mantuvieron acordes para los requerimientos de la especie (Figura 7).

En cuanto al peso inicial de las larvas no se observaron diferencias estadísticas significativas $(P>0.05)$; sin embargo, en el incremento final del peso sí se observaron diferencias significativas entre los tratamientos evaluados $(\mathrm{P}<0.05)$. Los cistos de Artemia son los que menor incremento diario presentaron (Tabla 2).

Con respecto a la Tasa Especifica de Crecimiento (TEC), comparado con los demás tratamientos, el mejor desempeño de las larvas se observó en los individuos alimentados con Nauplios de Artemia (20.34

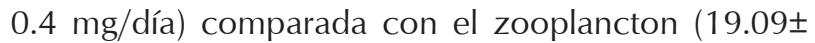
$0.42 \mathrm{mg} /$ día) y los cistos de Artemia (16.54 $\pm 0.34 \mathrm{mg} /$ día).

Los porcentajes de sobrevivencia fueron considerablemente mayores en los nauplios de artemia y zooplanc-

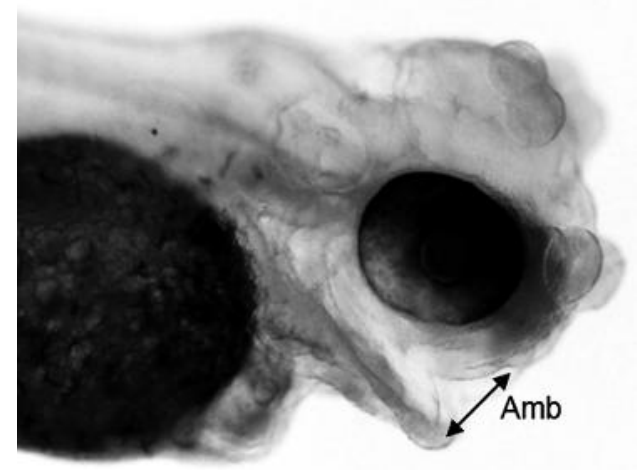

Figura 6. Abertura máxima bucal (Amb) de larva de P.altum de 75 HPE (15X).

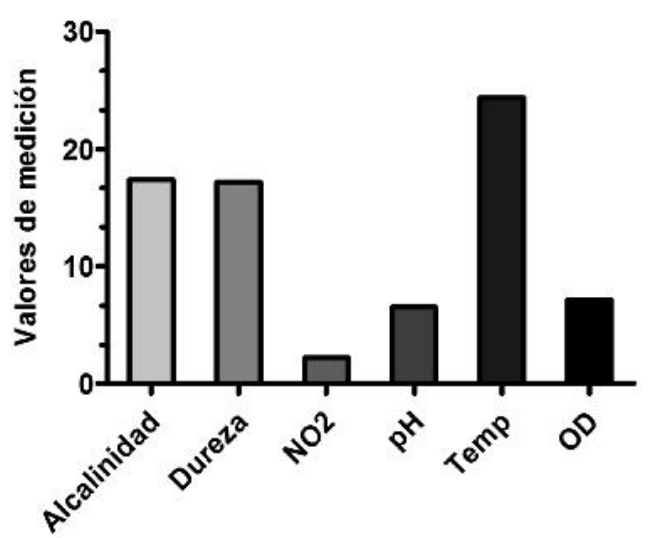

Figura 7. Parámetros físico-químicos del agua en acuario donde permanecieron las larvas de $P$. altum durante la fase de alimentación.

ton con una diferencia de un $52 \%$ con respecto a los cistos de artemia.

\section{Discusión}

Los estudios biológicos sobre las características del desarrollo, crecimiento, alimentación, comportamiento y mortalidad de embriones, larvas y postlarvas, son de gran interés para determinar las características biológicas de las especies. Los resultados obtenidos en el presente estudio concuerdan con trabajos desarrollados por otros autores (Korzelecka-Orkisz et al., 2012).

Las larvas de $P$. altum de un día de vida exhibieron pigmentación a lo largo de su cuerpo con pigmentos muy pequeños en la zona media del cuerpo, así como la presencia de algunos primordios alargados que formarían los filamentos branquiales, los cuales durante el cuarto y quinto día, se abrirán para iniciar el intercambio de gases (Korzelecka et al., 2010). De acuerdo con Ciechomski (1981), las larvas de Pterophyllum scalare son transparentes durante sus primeros días de vida,

Tabla 2. Índices de crecimiento de larvas de escalar P. altum. Datos mostrados como promedio \pm desviación estándar.

\begin{tabular}{|l|c|c|c|}
\hline \multirow{2}{*}{ Índices de crecimiento } & \multicolumn{3}{|c|}{ Tratamientos } \\
\cline { 2 - 4 } & Nauplios de artemia & Cistos de artemia & Zooplancton Silvestre \\
\hline Peso inicial (mg) & $2.05 \pm 0.00^{\mathrm{a}}$ & $2.05 \pm 0.00^{\mathrm{a}}$ & $2.05 \pm 0.00^{\mathrm{a}}$ \\
\hline Peso final (mg) & $58.50 \pm 4.12^{\mathrm{a}}$ & $28.50 \pm 5.34^{\mathrm{c}}$ & $48.50 \pm 3.74^{\mathrm{b}}$ \\
\hline Incremento diario (mg/dia) & $3.9 \pm 0.15^{\mathrm{a}}$ & $1.9 \pm 0.25^{\mathrm{c}}$ & $3.23 \pm 0.12^{\mathrm{b}}$ \\
\hline TCE (mg/dia) & $20.34 \pm 0.4^{\mathrm{a}}$ & $16.54 \pm 0.34^{\mathrm{c}}$ & $19.09 \pm 0.42^{\mathrm{b}}$ \\
\hline Sobrevivencia (\%) & $85.00 \pm 12.10^{\mathrm{a}}$ & $45.00 \pm 10.00^{\mathrm{b}}$ & $85.00 \pm 10.0^{\mathrm{a}}$ \\
\hline
\end{tabular}

Tasa Específica de Crecimiento ( $\mathrm{TEC}=\%$ peso corporal ganado mg/día). $\mathrm{T} 1=$ Nauplios de artemia. $\mathrm{T} 2=$ Cistos de artemia. $\mathrm{T} 3=$ Zooplancton Silvestre . Valores en la fila que no comparten el mismo superíndice son significativamente diferentes $(P<0.05)$. 
como estrategia de camuflaje y ser invisibles para los predadores.

En trabajos realizados por Korzelecka-Orkisz et al. (2010) en larvas de escalar, encontraron características morfológicas similares a lo observado en el presente estudio. Las larvas recién eclosionadas median en promedio $2.60 \pm 0.09 \mathrm{~mm}$. El tamaño de sus sacos vitelinos oscilaba en promedio $0.64 \pm 0.07 \mathrm{~mm}^{3}$ y disminuía de tamaño en proporción directa al crecimiento de la larva.

Los cambios observados entre LT y LS de larvas de 0-3 días fueron de tipo alométrico, dimensiones que fueron características durante todo su desarrollo. Estas mediciones concuerdan con trabajos desarrollados por Matsuura (1975) y Fahay (1983). De igual forma, la disminución en la altura de las larvas viene secuenciada de la reabsorción del saco vitelino, coincidiendo con la mayor mortalidad de larvas. En los peces, la transición de larvas con saco vitelino a larvas en la primera alimentación puede causar una mortalidad diaria de 26 al 97\% (Leaky Houde, 1987; Dorsey et al., 1996).

La duración del saco vitelino es un factor importante para la supervivencia larval, debido a que al completarse la alimentación lecitotrófica y comenzar la alimentación planctotrófica la larva exhibe características propias como pigmentación ocular y desplazamiento para captura de alimento y evitar la -predación (Miranda et al., 1992). Por tal razón, las larvas de peces que reabsorban su saco vitelino durante un período de tiempo prolongado, estarán expuestos a los riesgos de predación (Van Der Lingen, 1994).

Cuando las reservas vitelinas de la larva son completamente utilizadas, entran en un periodo crítico de alimentación planctotrófica; por consiguiente aumenta la mortalidad entre la fase postlarval y la fase larvaria. Tal como se ha observado en estudios previos, la mortalidad en peces durante los primeros estadios es mucho mayor que en cualquier estado de desarrollo (Beyery Laurence, 1980; Gómez, 1984; Van Der Lingen, 1994).

El crecimiento en longitud se mantuvo desde la eclosión hasta el segundo día de vida de la larva, para luego experimentar un decrecimiento el tercer día de vida. Probablemente este fenómeno sea originado por varios factores como la preflexión del notocordio. Van Der Lingen (1994) señaló cambios relativamente pequeños que preceden a la transformación. Durante la preflexión del notocordio se reducen las medidas del cuerpo por elevación del mismo. A su vez, la inanición de las larvas puede resultar en una disminución o con- tracción de tejidos blandos, como el tracto digestivo y algunas glándulas en formación, que son los primeros tejidos afectados (Theilacker, 1978).

Las fases de crecimiento observadas en el presente estudio (tres fases) donde la tercera presenta un crecimiento negativo, también ha sido reportada en otras especies de agua dulce y marina (Hunter y Kimbrell, 1980); Lates calarifer (Konho, et al., 1986); Acanthopagrusschlegeli (Fukuhara, 1987); Dichistiuscapensis (Van der Lingen, 1994). Esta disminución en la altura de las larvas viene secuenciada de la reabsorción del saco vitelino, coincidiendo con la mayor mortalidad de larvas.

El análisis de la morfometría y merística ha sido ampliamente utilizado con resultados satisfactorios, principalmente en la determinación de procesos microevolutivos en varias especies de importancia comercial (George-Nascimento y Arancibia 1992). Estudios desarrollados en escalares por Korzelecka-Orkisz et al. (2012) muestran que el número de miomeros se halla directamente relacionado con el desarrollo de las larvas y su estado de pigmentación, haciendo que sean más visibles. Los miomeros conforman la musculatura del tronco conformada por una serie de bloques musculares, separados por vainas de tejido conectivo Ilamados mioseptos. Estos mioseptos son representativos de la segmentación corporal de los embriones y larvas.

La nutrición de las larvas de peces es uno de los principales factores limitantes para la producción acuícola (Gore, 2006). La piscicultura de peces ornamentales no es la excepción, aún más cuando son especies que tienen una alta demanda a nivel mundial como los escalares. Los nauplios de Artemia son el alimento vivo más utilizado en el cultivo de numerosas especies acuáticas (Sarma et al., 2003) y el que mejores resultados arrojo en el presente estudio (Tabla 2). Sin embargo, existen diferentes inconvenientes con este producto, debido principalmente a su alto costo a nivel comercial y su variable calidad nutricional (Léger et al., 1986). Por tal motivo se ha creado la necesidad de realizar ensayos en la búsqueda de cultivos alternativos que suplan los requerimientos nutricionales de las diferentes larvas de peces. En esta búsqueda, cepas de zooplancton como los cladóceros, rotíferos y copépodos se han convertido en el alimento vivo más común para la alimentación de organismos en sus primeros estadios larvarios (Lim et al., 2001). De acuerdo con los estudios realizados por Nandini y Sarma (2000) y Sarma et al. (2003), las crías de $P$. altum, a partir de la segunda semana de vida, prefieren en su dieta cladóceros que rotíferos, postulados que concuerdan con los resultados del presente estudio. 
Así mismo, Figueroa et al. (2010) demostraron que larvas de $P$. scalare tenían un aumento mayor de peso al ser alimentadas con Artemia salina y que juveniles de la misma especie tenían un mayor rendimiento al ser alimentados con Moina sp. Por otro lado la alimentación artificial como el concentrado con sus diferentes porcentajes de proteína y presentaciones tienen unos resultados bajos al ser utilizados en estados iniciales de desarrollo larval debido a su baja digestibilidad (García-Ortega, 2000). Así mismo, podemos observar (Tabla 2.) que los cistos de artemia tienen bajos valores en las variables medidas comparados con los demás tratamientos debido probablemente a la baja digestibilidad al no estar decapsulados (Figueroa et al., 2010) lo cual influyó de manera negativa en los porcentajes de sobrevivencia de los individuos alimentados con estos microorganismos. Así mismo, según Figueroa et al. (2010) los nauplios tienen mayor digestibilidad de sus compuestos proteicos y lipídicos (57.26\%) comparado con $16.2 \%$ de cistos de artemia.

Los porcentajes de sobrevivencia fueron considerablemente mayores en los nauplios de artemia y zooplancton con una diferencia de un $52 \%$ con respecto a los cistos de artemia esto debido a la baja digestibilidad de los cistos los cuales pueden generar constipación y posterior muerte de las larvas. En investigaciones realizadas en $P$. scalare, se han obtenido porcentajes de sobrevivencia similares a los del presente estudio, dentro de un intervalo que va de 60 a 91\% (Ortega-Salas et al., 2009) o iguales, con 100\% de sobrevivencia (Rodrígues y Fernandes, 2006; Luna-Figueroa et al., 2007).

Los individuos de $P$. altum alcanzan su talla comercial en tiempo relativamente corto (60 y 70 días) por lo tanto los altos porcentajes de sobrevivencia son importantes por su alta demanda a nivel comercial.

Esta información constituye un aporte hacia la obtención e identificación de mecanismos o estrategias que garanticen una mayor eficiencia en la producción en cautiverio de $P$. altum, de acuerdo a las características morfológicas, morfométricas y merísticas determinadas en el presente estudio, así como el mejor desempeño productivo en individuos alimentados con nauplios de artemia, constituyéndose en una importante alternativa como fuente de alimento durante la larvicultura de esta especie.

\section{Agradecimientos}

Los autores agradecen al MVZ Andrés Arévalo Arroyave, a la Universidad de los Llanos y al Instituto de Acuicultura de los Llanos (IALL) por su apoyo logístico.

\section{Referencias}

Abdolbaghian S, Jamili S, Matinfar A, The effect of temperature and diet on the degrees of specific growth rate percentage (SGR $\%$ ) and weight growth (WG \%) of angel fish fry Pterophyllum scalare. J Fish Aquat Sci. 2010;5:311-315.

Agudelo DA. Establecimiento de un centro de reproducción de Pterophyllumscalare (pez ángel o escalar) Revista la Sallista de Investigación. 2006;2(2):26-30.

Axelrod H, Walker B. 2000. The guide to owning angelfish. New Jersey, TFH Publication, Inc., 64p.

Beyer J, Laurence G. A, Stochastic model of larval fish growth. Ecol Model. 1980;8:109-132.

Britski H, Sato A, Rosa AB. 1988. Manual de identificação de peixes da região de TresMarías (com chaves de identificação para os peixes da bacia do São Francisco). Brasilia: Cãmara dos Deputados, Coordenação de Publicaçoes CODEVASF.

Castro DM. Fauna acuática de la Amazonia Colombiana. En: Primer encuentro nacional de investigadores de la Amazonia. Colciencias, ICFES, Universidad de la Amazonia. Serie memorias y eventos científicos colombianos. 1985;38:75-82.

Ciechomski J. 1981. Ictioplancton. En: Atlas de zooplancton del Atlántico sudoccidental y métodos de trabajo con el zooplancton marino. Boltovsky, D. (Ed.). Publicación especial del INIDEP, Mar de Plata, Argentina. pp. 829-860.

Ditty CJ, Houde E, Shaw R. Egg and larval development of spanish sardine, Sardinella aurita (Family Clupeidae), with a synopsis of characters to identify clupeid larvae from the northern Gulf of Mexico. Bull Mar Sci. 1994;54(2):367-380.

Erdogan F, Olmez M. Effects of enzyme supplementation in diets on growth and feed utilization in angel fish, Pterophyllum scalare. J Anim Vet. 2009;8(8):1660-1665.

Fahay MP. 1983. Guide to the early stages of marine fishes occuring in the western North Atlantic Ocean, Cape Hatteras to the Southern Scotian Shelf. Journal of the Northwest Atlantic Fisheries Society 4, 423.

Fukuhara O. Larval development and behavior in early life stages of Black Sea bream reared in the laboratory. Bull. Japan Soc Scient Fish. 1987;53(3):371-379.

García-Ortega A. 2000. Valor nutricional de los quistes de Artemia y su uso como fuente de proteína en dietas artificiales para larvas de peces. En: Cruz-Suárez, L. E.; D. Ricque-Marie; M. Tapia-Salazar; M. A. Olvera-Novoa y R. Civera-Cerecedo (Eds.). Avances en nutrición acuícola V. Memorias del V Simposium Internacional de Nutrición Acuícola. 19-22 noviembre, 2000. Mérida, Yucatán, México. pp. 287-299.

George-Nascimento M, Arancibia H. Stocks ecológicos del jurel (Trachurus symmetricus murphyi Nichols) en tres zonas de pesca frente a Chile, detectados mediante comparación de su fauna parasitaria y morfometría. Rev Chil Hist Nat. 1992;65:453-470.

Glencross BD, Booth M, Allan GL. A feed is only as good as its ingredients a review of ingredient evaluation strategies for aquaculture feeds. Aquacult Nutr. 2007;13:17-34. 
Godinho HP, Santos JE, Sato Y. 2003. Ontogenesis larval de cinco espécies de peixes do Sao Francisco. En H. P. Godinho, \& A. L. Godinho, Águas. peixes e pescadores do Sao Francisco das Minas Gerail Belo Horizonte: PUC Minas. pág. 468

Gore RS. Nutritional support of fish. J Exot Pet Med. 2006; 15(4):264268.

Guillaume J, Kaushik S, Bergot P, Métailler R. 2004. Nutrición y alimentación de peces y crustáceos. Ediciones Mundi-Prensa. Madrid, España. 475 pp.

Halver JE. 1988. Fish Nutrition. Academic Press, Inc. School of Fisheries, University of Washington, Seattle, Washington. New York. 798 pp.

Hamre K, Srivastava A, Ronnestadl, Mangor-Jensen A, Stoss J. Several micronutrients in the rotifer Brachionus sp. may not fulfil nutritional requirements of marine fish larvae. Aquacult Nutr. 2008;14:51-60.

Hunter J, Kimbrell C. Early life history of Pacific mackarel, Scomber japonicus. Fish Bull Wash. 1980;78(1):89-101.

Kohno H, Hara S, Taki Y. early larval development of the seabass Lates calcarifer with emphasis on the transition of energy sources. Bull. Japan. Soc Scient Fish. 1986;52(10):1719-1725.

Korzelecka-Orkisz A, Smarujl, Pawlos D, Robakowski P, Tañski A. Szulc J, Formicki K. Embryogenesis of the stinging catfish, Heteropneustes fossilis (Actinopterygii: Siluriformes: Heteropneustidae). Acta Ichthyol Piscat 2010;40:187-197.

Korzelecka-Orkisz A, Smaruj I, Pawlos D, Robakowski P, Tañski A, Szulc J,Formicki K. Early ontogenesis of the angelfish, Pterophyllum scalare Schultze, 1823 (Cichlidae) Neotrop Ichthyol. 2012; 10(3):567-576.

Leak J, Houde E. Cohort growth and survival of bay anchovy Anchoamitchilli larvae in Biscayne Bay, Florida. Mar Ecol Prog Ser. 1987;37:109-122.

Léger P, Bengtson D, Simpson KL, Sorgeloos P. The use and nutritional value of Artemia as a food source. Oceanogr. Mar. Biol Ann Rev. 1986;24:521-623.

Luna-Figueroa J. Nematodo de vida libre Panagrellusredivivus (Goodey, 1945): Una alternativa para la alimentación inicial de larvas de peces y crustáceos. Investig Cienc. 2009;45:4-11.

Matsuura Y. A Study of the life history of Brazilian sardines, Sardinella brasiliensis. III. Development of sardine larvae. Bol. Inst. Oceanogr. S. Paulo. 1975;23:17-29.

Miranda A, Sánchez J, Álvarez F, Alemany F. Crecimiento larvario de sardina Sardinapilcharchurdus en laboratorio bajo diferentes condiciones de alimentación. Informes Téc. Inst. Esp. Oceanogr. 1992;132:1-15.

Nakatani K, Agostinho AA, Baumgartner G. 2001. Ovos e larvas de peixes de água doce: desenvolvimiento e manual de identificaçao. Maringá: EDEUM.

Nandini S, Sarma SS. Zooplankton preference of two species of freshwater ornamental fish larvae. J Appllchthyol. 2000;16:282284.

Norman Jr. 1975. A history of Fishers. 3ra ed.London. Ernest Been Limited. 467 p.

Ortega-Sala AA, Cortés GI, Reyes-Bustamante H. Fecundity, growth and survival of the angel fish Pterophyllums calare (Perciformes: Cichlidae) under laboratory conditions. Rev Biol Trop. 2009;57(3):741-747.

Pérez E, Díaz F, Espinac S. Thermoregulator y behavior and critical therma llimits of the angel fish Pterophyllums calare (Lichtenstein) (Pisces: Cichlidae). J Therm Biol. 2003; 28:531-537

Rodrigues LA, Fernandes JB. Inflúencia do processamento da dieta no desempenho produtivo do acará bandeira (Pterophyllums scalare). Acta Sci. Anim Sci. 2006;28(1):113-119.

Sales J, Janssens GP. Nutrient requirements of ornamental fish. Aquat Living Resour. 2003;16:533-540.

Sarma SS, López-Rómulo JA, Nandini S. Larval feeding behavior of blind fish Astyanax fasciatus (Characidae), black tetra Gymnocorym busterntzi (Characidae) and angel fish Pterophyllum scalare (Cichlidae) fed zooplankton. Hydrobiologia. 2003;510:207216.

Shirota A. Studies on the mouth size of fish larvae. Bull. Jpn. Soc. Sci. Fish. Tokyo. 1970;36:353-368.

Sorgeloos P, Lavens P, Léger P, Tackaert W. 1991. State of the art in larviculture of fish and shellfish. En P. Lavens, P. Sorgeloos, E. Jaaspers, \& F. Ollevier, Larvae Fish and Cruatacean Larviculture Symposium (Vol. 15, págs. 3-5). Gent, belgium: Europen Aquaculture Society.

Theilacker G. Effect of starvation on the histological and morphological charasteristics of jack mackerel, Trachurus symmetricus, larvae. Fish Bull. 1978;76(2): 403-414.

Van Der Lingen C. Aspects of the early life history of galjo in Dichistius capensis. S Afr. J mar Sci. 1994:14:37-45.

Zuanon JAS, Salaro AL, Balbino EM, Saraiva A, Quadros M, Fontanari RL. Níveis de proteína bruta em dietas para alevinos de acará-bandeira Pterophyllum scalare. Rev Bra Zootec. 2006;35(5):1893-1896. 